\title{
EVALUATING PHOTOGRAMMETRIC APPROACH OF IMAGE-BASED POSITIONING
}

\author{
Xun Li, Jinling Wang \\ School of Surveying and Spatial Information Systems, \\ University of New South Wales \\ xun.li@student.unsw.edu.au
}

KEY WORDS: Photogrammetry, Close Range, Image, Camera, Orientation, Accuracy, Impact Analysis

\begin{abstract}
:
In recent years, researches in the domain of location-based services have increasingly focused on developing and utilizing alternative positioning techniques for in GPS-denied environment. Image based positioning technique holds good promise for such applications. In this paper, a previously proposed image-based positioning system using photogrammetric methods has been put into rigorous evaluation. The precision and accuracy of such photogrammetric approach of image-based positioning is depending on the precision and accuracy of final space resection process, which is a function of PGCP distribution and measurement accuracy, and any factor that has certain impact on either of these two major components will to certain degree influence final positioning accuracy. Therefore in this article, the way that different factors influencing the positioning accuracy are analysed through both mathematical model and experiments, which includes simulations and tests based on real data. Through evaluation of such system, we aims at better understanding image-based positioning system alike so as to find its strength, weaknesses and ways to improve the overall performance for it to realize its full potential.
\end{abstract}

\section{INTRODUCTION}

In recent years, researches in the domain of locationbased services have increasingly focused on developing and utilizing alternative positioning techniques that enable position information to be more widely achievable and easy to be deployed to common users. Vision based applications hold good promise in a way that it works well in GPS-denied environment and cameras, the only sensor it requires, have been widely adopted on mobile devices. Image-based positioning, which derived from appearance-based visual systems, determine the position and orientation of the imaging sensor by comparing the images/templates with its current view. It can be categorised into two groups: one that obtaining the query image position by referring to an image database and the other via the analysis of sequential images (e.g. Huang and Netravali 1994). Our method belongs to the first class. Traditional approach is to match the query image with the images in the database, whenever a match is found, the position information of this reference image is transferred directly to the query image and used as user position. The problem lies in that the relative position between query image and reference image is not considered, which leads to the loss of accuracy. The vague position being close to the scene of the reference image may be expanded with additional information on the relation of the query and reference images (e.g. Steinhoff et al. 2007). An improved approach is to make triangulation of three reference images to get query image position; however, three matched images from the database may not be found. An alternative approach proposed by Zhang et al (2006) is to interpolate between the two references views to calculate the position of query image, while still at least two images are required. Our image-based positioning method on the other hand, has no requirement for the number of reference images and the final positioning is based on least squares adjustment of space resection, both of which are developed in the hope to develop the image-based positioning further. The major difference lies in that the reference images from database are geo-referenced. In this paper, our previously proposed image-based positioning system using photogrammetric methods ( $\mathrm{Li}$ et al, 2011a) has been put into rigorous evaluation in order to gain an insight of the potential of this method, which is critical for achieving seamless localization for location-based services.

The development of the image-based positioning\& navigation system mainly consists of two steps: mapping and positioning\& navigation. First a mapping procedure is carried out. Images of the navigational environment are collected and SIFT matching (Lowe, 1999) between images with overlapped areas is performed. The aim is to produce geo-referenced images of the navigation environment. More specifically, SIFT feature points on map images will be geo-referenced through photogrammetric bundle adjustment (indirect georeferencing). Two major inputs of bundle adjustment are: ground control points from ground control survey and tie points produced by the previous SIFT matching process. The quality of the map depends on the accuracy of georeferencing. At the real time positioning stage, when real 
time images are taken by the vision sensor mounted on the (moving) vehicle, another image matching based on SIFT is carried out between the real time image and the map images. When any of the SIFT feature points from the map image(s) finds its correspondence on the real time image, the geo-information it carried can be transferred to its counterpart. Therefore, matched SIFT features on the real time image obtain both image coordinates from matching process and 3D coordinates from map images, which can later serve as pseudo ground control points (PGCPs) for space resection based positioning at the final stage. The robustness against errors and reliability aspect of the system has been taken care of by the system outlier detection mechanism ( $\mathrm{Li}$ et al, 2011b).

The precision and accuracy of such photogrammetric approach of image-based positioning is depending on the precision and accuracy of final space resection process, which is a function of PGCP distribution and measurement accuracy, and any factor that has certain impact on either of these two major components will to certain degree influence final positioning accuracy. Therefore in this article, the way that different factors influencing the positioning accuracy are analysed through both mathematical model and experiments, which includes simulations and tests based on real data.

The paper is constructed as follows: the first section introduces our designed image-based positioning system and such system in general; the second one identifies the two major components that determine final image-based positioning accuracy and proposes the way to evaluate them through both mathematical model and tests; in the following two sections, the impact from the two components, geometry and measurement are analysed respectively through real world dataset as well as simulations. we give our concluding remarks in the last section.

\section{MAJOR COMPONENTS DETERMINING POSITIONING ACCURACY}

In this section, we identify the two main components that determine the accuracy of the position solution: geometry and measurement accuracy. Both mathematical model and test results are analysed to verify this assumption. Any factor that involved impacts the positioning accuracy is through its influence on these two elements.

\subsection{Mathematical Models}

Space Resection is a photogrammetric method of solving single image orientation. The best known method for space resection is based on a least squares solution of linearised collinearity equations (Eq.1). It provides the highest level of accuracy with the presence of redundant measurements.

$$
\begin{aligned}
& x-(x)=-f \frac{a_{1}\left(X-X_{S}\right)+b_{1}\left(Y-Y_{s}\right)+c_{1}\left(Z-Z_{s}\right)}{a_{3}\left(X-X_{s}\right)+b_{3}\left(Y-Y_{s}\right)+c_{3}\left(Z-Z_{s}\right)} \\
& y-(y)=-f \frac{a_{2}\left(X-X_{S}\right)+b_{2}\left(Y-Y_{S}\right)+c_{2}\left(Z-Z_{s}\right)}{a_{3}\left(X-X_{s}\right)+b_{3}\left(Y-Y_{S}\right)+c_{3}\left(Z-Z_{s}\right)}
\end{aligned}
$$

Here we use least squares based space resection with modification for the final positioning resolution ( $\mathrm{Li}$ et al, 2011b). The matched SIFT feature points (on real time image) with geo-information transferred from its counterpart (on map images) served as ground control points(PGCP). Since the 3D object space coordinates of PGCPs are photogrammtrically determined, which are not accurate enough to be used as error-free reference and held fixed, they are introduced into the system as observed unknowns (pseudo observations) with a corresponding weight. The Gauss-Markov Model for space resection is as follows:

$$
A t+B X-l_{1}=v_{1}, \quad l_{1} \sim\left(0, \sigma_{0}^{2}{\mathbf{P}_{1}}^{-1}\right)
$$

in which $\boldsymbol{A}$ contains partial derivatives with respect to the exterior orientation parameters, and $t$ contains the incremental changes to the initial values of external orientation parameters; $\boldsymbol{B}$ contains the partial derivatives with respect to the three coordinates of the (Pseudo) Ground Control Points, and $\boldsymbol{X}$ contains the incremental changes to the initial values of ground coordinates of PGCP. It should be noted that it is still a multi-solution equation when geo-referencing information from ground coordinates is not available. Therefore, absolute orientation information needs to be introduced into the adjustment with stochastic constraints:

$$
I X-l_{2}=v_{2} \quad, \quad l_{2} \sim\left(0, \sigma_{0}^{2} \mathbf{P}_{2}^{-1}\right)
$$

Combine (2) with (3):

$$
\left[\begin{array}{ll}
A & B \\
0 & I
\end{array}\right]\left[\begin{array}{l}
t \\
X
\end{array}\right]-\left[\begin{array}{l}
l_{1} \\
l_{2}
\end{array}\right]=\left[\begin{array}{l}
v_{1} \\
v_{2}
\end{array}\right], \quad\left(\begin{array}{ll}
\mathrm{P}_{1} & 0 \\
0 & \mathrm{P}_{2}
\end{array}\right)
$$

Corresponding normal equation becomes:

$$
\begin{aligned}
{\left[\begin{array}{ll}
A & B \\
0 & I
\end{array}\right]^{T}\left(\begin{array}{ll}
\mathbf{P}_{1} & \mathbf{0} \\
\mathbf{0} & \mathbf{P}_{2}
\end{array}\right) } & {\left[\begin{array}{ll}
A & B \\
0 & I
\end{array}\right]\left[\begin{array}{l}
\boldsymbol{t} \\
X
\end{array}\right] } \\
& =\left[\begin{array}{ll}
A & B \\
0 & I
\end{array}\right]^{T}\left(\begin{array}{ll}
\mathbf{P}_{1} & \mathbf{0} \\
0 & \mathbf{P}_{2}
\end{array}\right)\left[\begin{array}{l}
l_{1} \\
l_{2}
\end{array}\right]
\end{aligned}
$$

Substituting some parts of the equation 5 with simple expression:

$$
\left[\begin{array}{ll}
N_{11} & N_{12} \\
N_{21} & N_{22}
\end{array}\right]\left[\begin{array}{l}
t \\
X
\end{array}\right]=\left[\begin{array}{l}
W_{1} \\
W_{2}
\end{array}\right]
$$

in which

$$
\left[\begin{array}{cc}
N_{11} & N_{12} \\
N_{21} & N_{22}
\end{array}\right]=\left[\begin{array}{cc}
A^{T} P_{1} A & A^{T} P_{1} B \\
B^{T} P_{1} A & B^{T} P_{1} B+P_{2}
\end{array}\right]
$$

The covariance matrix of the unknowns is contained in a generalized inverse of the normal equation matrix: 


$$
\left[\begin{array}{ll}
Q_{t t} & Q_{t X} \\
Q_{X t} & Q_{X X}
\end{array}\right]=\left[\begin{array}{ll}
N_{11} & N_{12} \\
N_{21} & N_{22}
\end{array}\right]^{-1}
$$

Since the quality of space resection based positioning is evaluated by the precision \&accuracy of 6DOF, a relative precision can be measured through the post-adjustment covariance matrix $\boldsymbol{Q}_{\boldsymbol{t} t}$ of the estimated image orientation in $6 \mathrm{DOF}(\boldsymbol{t})$, regarding $\sigma_{0}^{2}$ as a scale factor.

$$
Q_{t t}=\left(N_{11}-N_{12} N_{22}^{-1} N_{21}\right)^{-1}
$$

From Eq. (7)

$Q_{t t}=\left(A^{T} P_{1} A-A^{T} P_{1} B\left(B^{T} P_{1} B+P_{2}\right)^{-1} B^{T} P_{1} A\right)^{-1}$

From Eq. (10) we can clearly observe that the final positioning precision \&accuracy using a modified space resection model is affected by two major elements: geometry $(\boldsymbol{A} \& \boldsymbol{B})$ and the accuracy of measurements: image measurement ( $\left.\mathbf{P}_{\mathbf{1}}\right)$ and $3 \mathrm{D}$ object space coordinates of Pseudo Ground Control Points $\left(\mathbf{P}_{\mathbf{2}}\right)$.

In order to further investigate the role of the two components, their influence on the final precision need to be separately evaluated, we propose the use of DOP values as the indicator of geometric strength and the estimated standard deviation of observations to evaluate measurement accuracy. The calculation of DOP values follows the same way as in the GPS community

$$
\boldsymbol{C}_{\boldsymbol{X}}=\sigma_{0}^{2}\left(\boldsymbol{A}^{\boldsymbol{T}} \boldsymbol{A}\right)^{-1}
$$

in which the part $\left(\boldsymbol{A}^{\boldsymbol{T}} \boldsymbol{A}\right)^{-1}$ contains DOP factors in its diagonal elements.

The estimated standard deviation of observations is calculated as follows:

$$
\hat{\sigma}_{0}=\sqrt{\frac{\boldsymbol{v}^{T} \boldsymbol{P} \boldsymbol{v}}{f}}
$$

in which $\mathrm{f}$ represents degree of freedom $(\mathrm{f}=\mathrm{n}-\mathrm{u})$.

\subsection{Experiment Results with the Proposed Methodology}

In this experiment, real time images are obtained through stable camera stations rather than camera mounted on a moving vehicle. A calibrated CCD camera (Canon EOS4500) is used. In this way, each position of the camera site can not only be calculated and evaluated within our system but also measured by external tools (e.g. total station) with relatively higher accuracy. The positioning accuracy can be evaluated against reality.

According to Table 1, the accuracy of the positioning is between 1-10 centimetre level. So we analyse the estimated precision, the impact of geometry and measurement accuracy on them. Compare Fig.1 and
Fig.2, it can be observed that the position precision generally follows the trend of DOP, which means geometry has the biggest impact. At the same time, it is noted that they are not exactly the same: e.g. the precision on $\mathrm{Z}$ axis drops from epoch 3 to 4 , while their DOP values are close. There are only two components that contributing to the final precision: geometry and measurement accuracy. As shown in figure 3, the measurement accuracy from epoch 3 to 4 actually drops as predicted. Although the influence of measurement accuracy is not significant in this case, it still proves the point that the overall positioning precision and accuracy depend on geometry and measurement accuracy. And geometry is the major impact since the final image-based positioning uses the same image-matching algorithm and geo-referenced map, which means the measurement accuracy remains relatively stable. The geo-referencing accuracy of the map in different areas may varies, which leads to the variations on measurement accuracy.

\begin{tabular}{|c|c|c|c|c|}
\hline & $\begin{array}{c}\text { Camera } \\
\text { Site ID }\end{array}$ & $\mathrm{X}$ & $\mathrm{Y}$ & $\mathrm{Z}$ \\
\hline \multirow{4}{*}{ Calculated(m) } & 3 & 0.057 & 1.177279 & -1.27276 \\
\cline { 2 - 5 } & 4 & 0.07 & 1.843871 & -1.31133 \\
\cline { 2 - 5 } & 5 & 0.067 & 2.923681 & -1.26144 \\
\hline \multirow{4}{*}{\begin{tabular}{c} 
Surveyed(m) \\
\cline { 2 - 5 }
\end{tabular}} & 6 & 0.075 & 5.376772 & -1.27968 \\
\cline { 2 - 5 } Absolute & 3 & 0.0666 & 1.08 & -1.264 \\
\cline { 2 - 5 } $\begin{array}{c}\text { Difference } \\
\text { (m) }\end{array}$ & 5 & 0.0675 & 1.8851 & -1.2654 \\
\cline { 2 - 5 } & 3 & 0.0623 & 2.9549 & -1.2635 \\
\cline { 2 - 5 } & 5 & 0.0636 & 5.0033 & -1.264 \\
\cline { 2 - 5 } & 6 & 0.0025 & 0.097279 & 0.008755 \\
\hline \multirow{2}{*}{ Table 1. System } & 0.0047 & 0.031219 & 0.045929 \\
\hline
\end{tabular}

Table 1. System measured results evaluated against total station results

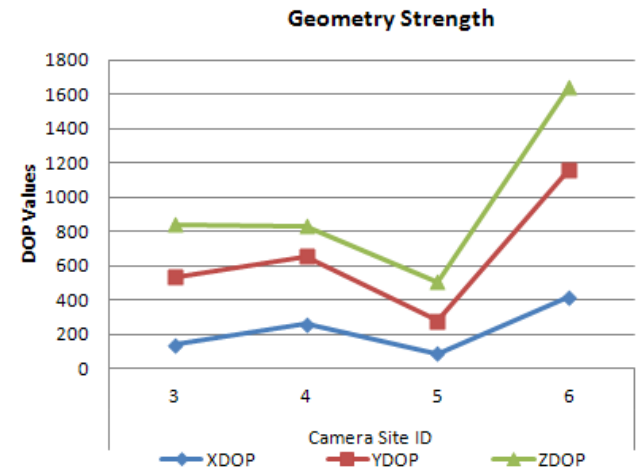

Figure 1.Geometric Strength on the 4 epochs Positioning Precision

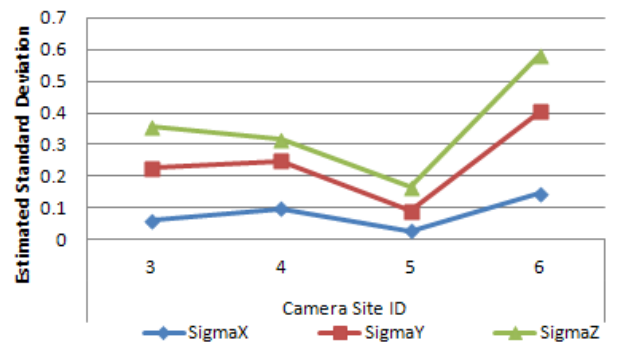

Figure 2. Positioning precision using estimated standard deviation in 3 out of 6 unknown parameters 


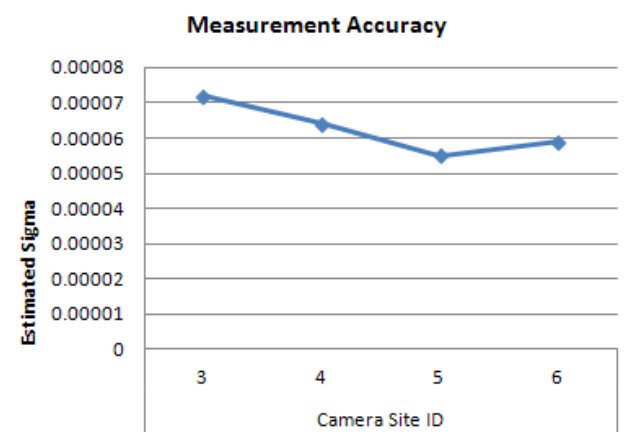

Figure 3. Measurement accuracy on the 4 epochs

\section{GEOMETRY AND FACTORS INVOLVED}

In this section, the major components that determines positioning accuracy, geometric configuration of PGCPs, are analysed.

\subsection{Geometric Impact}

This experiment aims at evaluating the geometric impact on final positioning accuracy. Image-based positioning is carried out in the same mapped indoor area. A calibrated video camera (Logitech Webcam Pro2000) is mounted on a moving vehicle with sampling rate at $1 \mathrm{HZ}$. Its relative position to the vehicle is fixed, which means the experiment is partially controlled: camera height (Z:$0.725 \mathrm{~m})$ and two angles of the camera attitude $(\omega=$ $1.57 \mathrm{rad}, \varphi=0 \mathrm{rad}$ ) are fixed. We do the positioning by extracting image frames from the video and match with the 3D map images frame by frame. Each frame is an epoch; a position in 6DOF is calculated. We took epoch No.20-40 with controlled parameter Z for illustration.

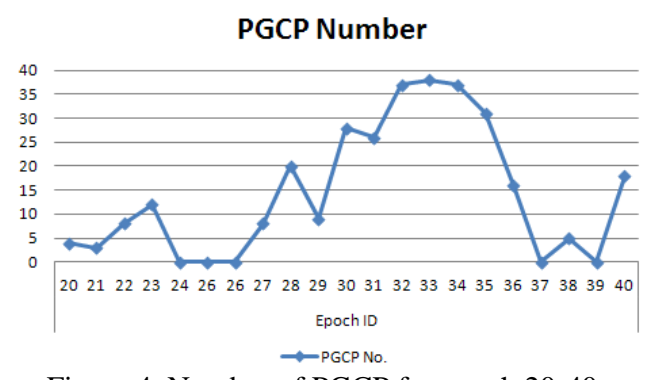

Figure 4. Number of PGCP for epoch 20-40

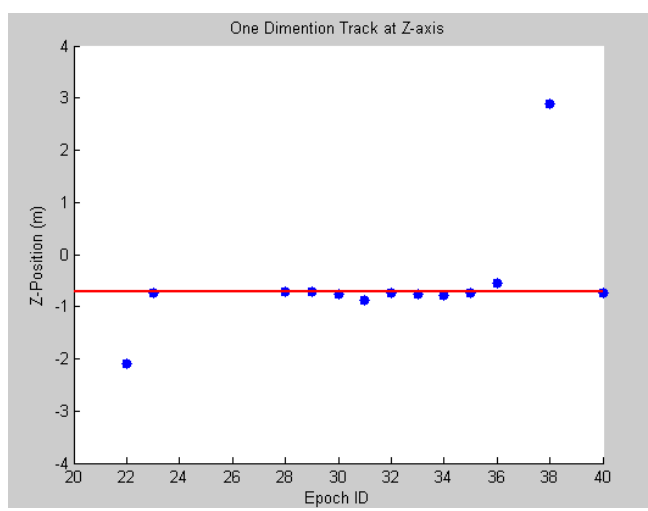

Figure 5. Measured Z position for epoch 20-40

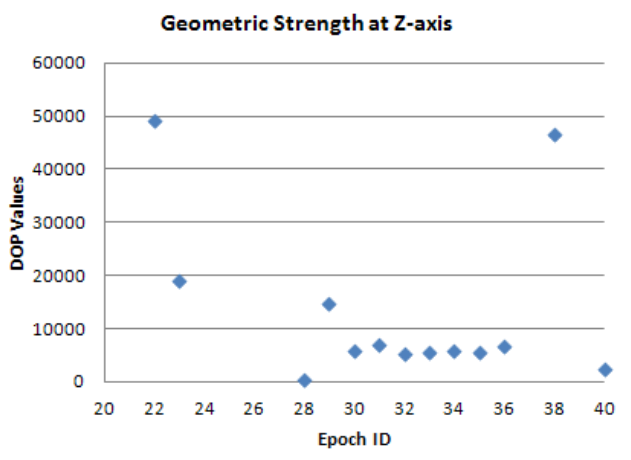

Figure 6. Geometric strength at Z for epoch 20-40

Fig. 5 shows the calculated $\mathrm{Z}$ position between epochs 20-40. In extreme cases, as shown in Figure 4\&5, if too few PGCPs are generated, the positioning calculation will fail. Compare Fig. 5 with the DOP values at Z-axis (Fig. 6), it can be clearly seen that big DOP values, which means bad geometry, are behind the bad positioning results with low accuracy (e.g. Epoch 22\&38). The rest of the results are reasonable while their DOP values are below a certain limit. And the absolute accuracy does not follow the exact trend of DOP. Put the 3 figures together, it's not hard to observe that a bigger number of PGCPs gives a better chance of good geometry, thus a more accurate positioning result, vice verse. Therefore, it is concluded that the major cause of inaccurate results is bad geometry, and geometric impact plays an important role in the determination of final positioning precision.

PGCPs are produced by matched SIFT feature points, therefore, any factor that influence the SIFT matching between query image and reference image(s) will affect the density and geometric configuration of PGCPs, which includes the richness of features, illumination, viewing angle, etc. In fact it is a common problem for vision-based systems for the positioning function depends heavily on the recognition of visual features. One direction is to develop more robust descriptor or image matching techniques for systems alike.

\section{MEASUREMENT ACCURACY AND FACTORS INVOLVED}

In this section, the second component that determines final positioning accuracy, measurement accuracy, is discussed. It is the overall accuracy indicator of observations in the system, which mainly comes from two groups: image measurements (the image coordinates of PGCPs) and 3D coordinates of PGCPs. The first group of observations are produced by SIFT feature extraction that have consistent accuracy, and the ground coordinates of PGCPs are provided by indirect geo-referencing.

In order to investigate how the accuracy of this two groups of observations influence measurement accuracy 
which further affect final accuracy of a position solution, Monte Carlo simulation is used. Monte Carlo simulation is a well proven and efficient way to investigate the numerical properties of a complex mathematical model with respect to artificial noise in the input data (Robert and Casella, 2002). Noise is added with due regard to statistical distributions and typical noise levels so that the resulting output data varies realistically (Luhmann, 2009). Here, Monte Carlo simulation is used to add noise to these two groups of observations respectively, and final positioning results along with statistical analysis report are calculated. The formula follows:

$$
\mathrm{P}_{\mathrm{m}}=\mathrm{P}_{0}+\left(\mathrm{nRNG} * \mathrm{~s}_{\mathrm{p}}\right)
$$

With $\quad \mathrm{P}_{\mathrm{m}}=$ randomly modified paramete; $\quad \mathrm{P}_{0}=$ input value of parameter $\mathrm{P}$; $\quad \mathrm{nRNG}=$ random value that follows standard normal distribution; $\quad s_{p}=$ standard deviation/ noise level. Therefore, parameter $\mathrm{P}$ is modified by adding noise ( $\left.n R N G * s_{p}\right)$ at the level of $s_{p}$.

\subsection{Influence of Image Measurement Accuracy}

First image measurement noise was simulated and added to the input of image coordinates for final space resection. The original input image coordinates $\left(\mathrm{P}_{0}\right)$ obtained by SIFT matching algorithm have a measurement accuracy at $0.00004 \mathrm{~m}\left(\sigma_{0}\right)$, the noise level added to the input has been set varies from $0 \mathrm{~m}$ to $0.00008 \mathrm{~m}$ at an interval of $0.00001 \mathrm{~m}$. At each noise level, the simulation runs 5000 times. The measurement accuracy (Sigma estimated) is calculated using the mean value of results from the same noise level. Figure 7 proves measurement accuracy decreases with the increasing noise level at image coordinates, which means the image measurement accuracy affect the measurement accuracy positively. Figure 8 illustrates the variation trend of DOP values with the increasing noise level, and it remains at a stable certain value, which proves the geometry is not affected. In order to investigate the impact of image measurement noise on final positioning accuracy, the inner precision (standard deviation) of 6DOF measurement within the simulation results is calculated at each noise level. It proves that $6 \mathrm{DOF}$ precision decreases with decreasing image measurement accuracy (Figure 9\&10).

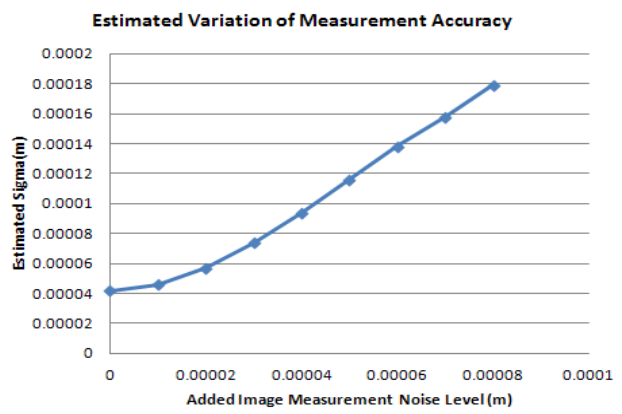

Figure 7. Variation of measurement accuracy at different image measurement noise level

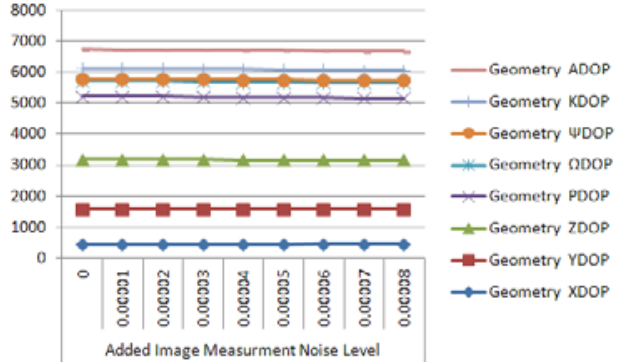

Figure 8. Geometric strength (DOP values in 6DOF) at different noise level

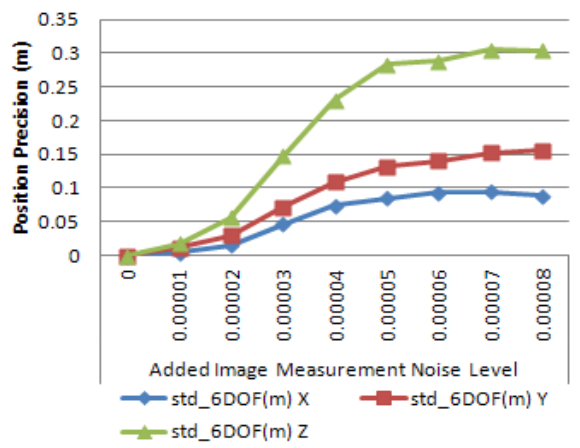

Figure 9. Variation of position precision at different image measurement noise level

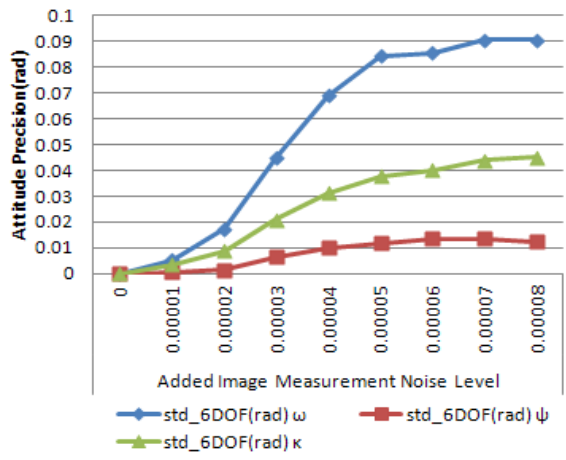

Figure 10. Variation of attitude precision at different image measurement noise level

\subsection{Influence of Geo-referencing Accuracy}

Another factor that influences the final positioning accuracy is the precision \& accuracy of 3D coordinates of PGCPs, which is determined by geo-referencing accuracy of the map images. In this experiment, noise is added to the 3D coordinates of PGCPs. The original input of PGCPs 3D coordinates have an accuracy at $0.03 \mathrm{~m}$, the noise level added to the input has been set varies from $0 \mathrm{~m}$ to $0.03 \mathrm{~m}$ at an interval of $0.005 \mathrm{~m}$. At each noise level, the simulation runs 5000 times. The measurement accuracy (sigma estimated) is calculated using the mean value of results from the same noise level. Figure 11 proves measurement accuracy decreases with the increasing noise level at object coordinates of PGCPs, which means geo-referencing accuracy of the map images affect the measurement accuracy positively. It is also observed that DOP values remain relatively stable with the increasing noise level, which means the geometry is not affected. 
In order to investigate the impact of 3D object coordinate noise on final positioning accuracy, the inner precision (standard deviation) of 6DOF measurement within the simulation results is calculated at each noise level. As shown in Figure 12 \&13, 6DOF precision decreases with decreasing 3D coordinates' accuracy. It can be observed that geo-referencing accuracy of the map images actually exerts certain amount of influence on final positioning accuracy via measurement accuracy. Therefore, in order to achieve a higher accuracy with such approach, the accuracy of bundle adjustment (indirect geo-referencing) need to be improved.

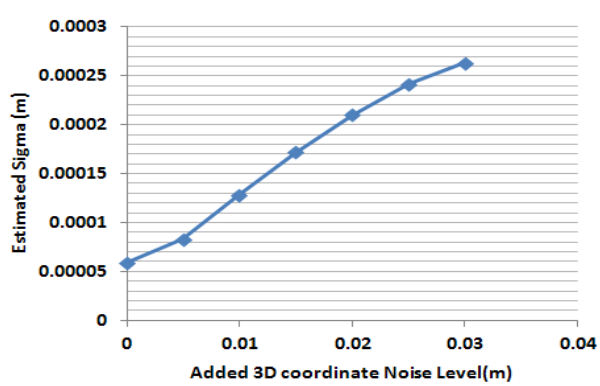

Figure 11. Variation of measurement accuracy at different noise level of 3D coordinates

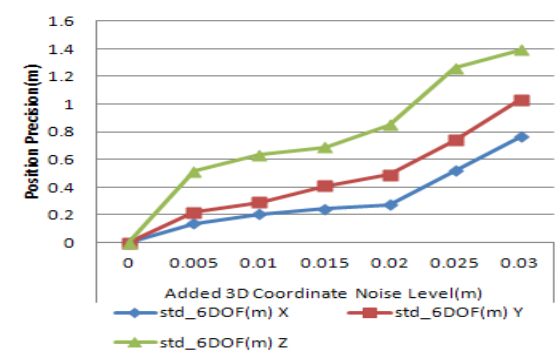

Figure 12. Variation of position precision at different noise level of 3D coordinates

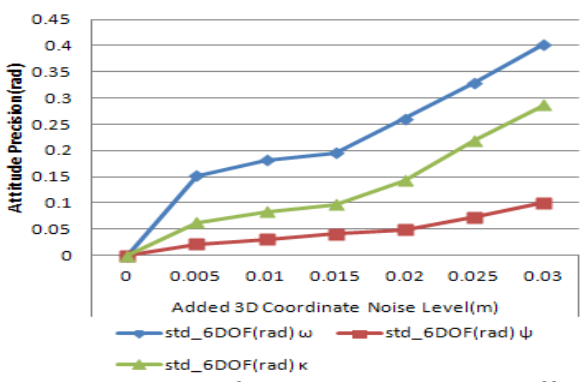

Figure 13. Variation of position precision at different noise level of $3 D$ coordinates

\section{CONCLUDING REMARKS}

In this paper, a previously proposed image-based positioning system has gone through a numerical evaluation with a focus on its accuracy. The accuracy of such positioning method is currently between 1-10 centimetre levels, which has not yet reach a satisfactory performance. Thus factors that affect its final accuracy are analysed, two major components: geometry of PGCP and measurement accuracy are identified. Geometric configuration of PGCP is closely related to the image matching procedure. Meanwhile, factors that influence measurement accuracy for final positioning are also identified and analysed: image measurement and georeferencing accuracy at mapping stage. Their influence is simulated by inserting artificial noise and the consequent impacts are evaluated by using Monte Carlo simulation. It is observed that the accuracy of map image georeferencing can exert a substantial effect on final positioning accuracy; therefore, future work will be focused on improving geo-referencing accuracy. At the same time, these two factors will be further evaluated against real data, and image matching technique used in the system will be further discussed and improved.

\section{REFERENCES}

C.P. Robert, G. Casella, 2002. Monte Carlo Statistical Methods, Springer, New York.

Cobzas, D., Zhang, H., and Jagersand, M. ,2003. Imagebased localization with depth-enhanced image map. Proceedings of the IEEE International Conference on Robotics and Automation (ICRA), pp. 1570-1575.

Huang, T. and Netravali, A., 1994. Motion and structure from feature correspondences: A review. Proceedings of IEEE, 82(2): 252-268.

Lowe, D.G., 1999. Object recognition from local scaleinvariant features. International Conference on Computer Vision, Corfu, Greece, pp. 1150 -1157.

Li X., Wang J., Yang L., 2011a. Outlier Detection for Indoor Vision-Based Navigation Applications. Proceedings of the 24th International Technical Meeting of The Satellite Division of the Institute of Navigation (ION GNSS 2011), Portland, OR, September 2011, pp. 3617-.

Li X., Wang J., Li R., Ding W., 2011b. Image-based positioning with the use of geo-referenced SIFT features. Proceedings of the Incorporating the International Symposium on GPS/GNSS (IGNSS 2011), Sydney, Australia, November 2011.

Steinhoff, U., Omercevic, D., Perko, R., Schiel, B., \& Leonardis, A. 2007. How computer vision can help in outdoor positioning. AmI 2007, LNCS 4794, pp. 124-141.

T. Luhmann, 2009. Precision potential of photogrammetric 6 DOF pose estimation with single images. ISPRS Journal of Photogrammetry and Remote Sensing, 64 (3) (2009), pp. 275-284

W. Zhang and J. Kosecka, 2006. Image based localization in urban environments. International Symposium on 3D Data Processing, Visualization and Transmission 